\title{
A PD-Type Iterative Learning Control for a Class of Switched Discrete-Time Systems with Model Uncertainties and External Noises
}

\author{
Xuan Yang \\ Department of Applied Mathematics, School of Mathematics and Statistics, Xian Jiaotong University, 28 Xianning West Road, \\ Xian, Shaanxi 710049, China \\ Correspondence should be addressed to Xuan Yang; yangxuan@stu.xjtu.edu.cn
}

Received 25 March 2015; Revised 27 May 2015; Accepted 9 June 2015

Academic Editor: Manuel De la Sen

Copyright (C) 2015 Xuan Yang. This is an open access article distributed under the Creative Commons Attribution License, which permits unrestricted use, distribution, and reproduction in any medium, provided the original work is properly cited.

\begin{abstract}
A PD-type iterative learning control algorithm is applied to a class of linear discrete-time switched systems for tracking desired trajectories. The application is based on assumption that the switched systems repetitively operate over a finite time interval and the switching rules are arbitrarily prespecified. By taking advantage of the super-vector approach, a sufficient condition of the monotone convergence of the algorithm is deduced when both the model uncertainties and the external noises are absent. Then the robust monotone convergence is analyzed when the model uncertainties are present and the robustness against the bounded external noises is discussed. The analysis manifests that the proposed PD-type iterative learning control algorithm is feasible and effective when it is imposed on the linear switched systems specified by the arbitrarily preset switching rules. The attached simulations support the feasibility and the effectiveness.
\end{abstract}

\section{Introduction}

A switched system consists of finite subsystems described by differential equations or difference equations and a switching rule which specifies a subsystem being activated during a certain time subinterval $[1,2]$. In the real world, the switched systems arise in many fields such as chemical processes, power systems, switched circuits, and traffic systems $[3,4]$. Thus investigation on the switched systems is necessary. It has drawn considerable attention since 1990s [5, 6]. In recent years, main efforts have been made on analyzing the dynamical behaviors of the switched systems such as stability $[1,4,7-$ 9], controllability, reachability [10,11], and observability [12]. It is emphasized that the switching rules play a significant role in the process of analyzing these dynamical behaviors $[2,13]$. For example, an improper switching may destabilize a switched system even if all subsystems are stable [2]. Conversely, an appropriate switching may stabilize a switched system even if its subsystems are unstable [2].

Control of the switched systems for some desirable performance is also a branch in the community of the switched systems research. There are many works on this issue. For instance, an approach for solving an optimal control problem of the switched systems has been presented in [14], where the prespecified sequences of the controlled systems are given. And, a kind of sliding mode control for the switched stochastic systems has been proposed in [15], where a sufficient condition is deduced to guarantee the mean-square exponential stability. Also, a finite time quantized $H_{\infty}$ control problem for the switched systems has been designed by solving an optimization problem in [16], where an average dwell time method and a mode-dependent quantized state feedback controller are employed.

Compared with the above stated issues, study on designing controllers for the switched systems to track given trajectories is relatively challenging $[3,4]$. Main reason may be that the arbitrary switching rules will result in indeterminate dynamical behaviors of the controlled switched systems, which does not meet requirement of trajectory tracking problem. Moreover, in engineering practice, the dynamics of the plant model is often unknown and various uncertain disturbances are unavoidable. Fortunately, for the repetitive systems, there exists a kind of efficient control strategy named iterative learning control (ILC). ILC has witnessed 
flourishing development in both theoretical analysis and practical application since its invention in [17]. ILC makes use of the tracking error information from previous or current trials to compensate for the current control input so as to generate an upgraded one for the next trial. Since it requires less knowledge of the system dynamics and spends less computational effort than other types of control methods [18], ILC is especially suitable for a kind of trajectory tracking control of the repetitive systems whose a priori knowledge is unknown and initial state can be periodically reset. In view of its nature properties, the ILC strategies can be applied to the switched systems meeting above requirement to track given trajectories.

However, according to ILC's mechanism, the activated subsystems running over a certain time subinterval in different iterations are required to be identical. This means that the dynamics of the controlled system is iteration-invariant. Thus, when the ILC strategies are used on the switched systems, a key assumption should be made that the switching rule which specifies activated subsystems at different time subintervals is also iteration-invariant. That is to say, once a switching rule is arbitrarily specified at the beginning iteration, it remains invariant during the subsequent learning process. Actually, provided that the switching rule is timevarying rather than iteration-varying, the above assumption can be easily satisfied. Using the above assumption, recent references $[3,13,18,19]$ have applied the ILC strategies to the switched systems.

In [3], a P-type ILC scheme has been applied to a class of linear discrete-time switched systems, where the convergence property is analyzed by the super-vector approach. In $[13,19]$, the D-type ILC algorithms have been used on a class of discrete-time linear switched systems and a kind of discretetime nonlinear switched systems, respectively. In addition, a high-order PID-type ILC scheme for a class of nonlinear switched systems with state delay has been analyzed from the perspective of stability in [18]. It is recalled that the learning performance analysis of the above ILC algorithms for the switched systems is mainly made in the sense of $\lambda$-norm which is helpful for deducing preferable theoretical results. However, it has been established that, in [20], analyzing the learning performance of an ILC algorithm in the sense of $\lambda$-norm is defective from the perspective of application. For example, the impact of the system dynamics and the learning gains will be extremely suppressed by a sufficiently large parameter $\lambda$ to ensure convergence. It may result in a situation that an improper choice of the parameter $\lambda$ would spoil a fair assessment of the tracking behaviors in practice [20]. In addition, [3, 13, 18, 19] do not involve robustness analysis when the controlled switched systems are disturbed by model uncertainties or external noises. However, both uncertainties and noises are inevitable in engineering practice. So, robustness is one of important considerations in the process of controller design. Analysis of an ILC algorithm regardless of robustness is inadequate from the perspective of practical application. In view of this, the study on the ILC algorithms for the switched systems needs to be refined or to be expanded.
Motivated by the above statement, we consider a PDtype ILC scheme for a class of linear discrete-time switched systems with arbitrarily prespecified switching rules. By using the super-vector approach, a sufficient condition of the monotone convergence is firstly deduced when both the model uncertainties and the external noises are absent. Using this deduced condition, the robust monotone convergence is analyzed when the model uncertainties are present and the robustness against the bounded external noises is discussed.

The rest of the paper is organized as follows. Section 2 presents the fundamental problem with respect to the switched systems and the ILC process. Section 3 exhibits the main analysis of the proposed PD-type ILC algorithm including the monotone convergence, the robust monotone convergence, and the robustness. In order to show the feasibility and the effectiveness of the algorithm, the numerical simulations are given in Section 4 and the conclusion is drawn in Section 5.

\section{Fundamental Problem Description}

Consider a class of uncertain linear discrete-time-invariant single-input, single-output switched systems described as follows:

$$
\begin{aligned}
\mathbf{x}_{k+1}(i+1)= & \left(\mathbf{A}_{\tau(i)}+\Delta \mathbf{A}(i)\right) \mathbf{x}_{k+1}(i) \\
& +\left(\mathbf{B}_{\tau(i)}+\Delta \mathbf{B}(i)\right)\left(u_{k+1}(i)+m_{k+1}(i)\right), \\
y_{k+1}(i+1)= & \mathbf{C}_{\tau(i)} \mathbf{x}_{k+1}(i+1)+n_{k+1}(i+1),
\end{aligned}
$$

$$
i \in S \text {. }
$$

Here, $S=\{0,1,2, \ldots, N-1\}, N \in \mathbb{Z}_{+}$stands for the operation time duration of each trial, $i \in S$ denotes sampling time instant, while $k+1, k \in \mathbb{N}$ represents iteration number. $\mathbf{x}_{k+1}(i) \in \mathbb{R}^{n}$ is state vector, $u_{k+1}(i) \in \mathbb{R}$ is control input signal, and $y_{k+1}(i+1) \in \mathbb{R}$ is system output. The symbol $\tau(i)$ represents the switching rule which is defined as $\tau(\cdot)$ : $\mathbb{N} \rightarrow Q=\{1,2,3, \ldots, q\}$ with $q \in \mathbb{Z}_{+}, q<+\infty$. The switching rule is piecewise constant function with respect to discrete-time $i$ and specifies which subsystem is activated at the time subinterval $[i, i+1)$. The constant matrices $\mathbf{A}_{\tau(i)}$, $\mathbf{B}_{\tau(i)}, \mathbf{C}_{\tau(i)}$ with appropriate dimensions are dynamics of the nominal system of (1). Obviously, specified by the definition of the switching rule, the matrices triplets $\left(\mathbf{A}_{\tau(i)}, \mathbf{B}_{\tau(i)}, \mathbf{C}_{\tau(i)}\right)$, $\tau(i) \in Q$, are elements of the following finite set:

$$
\left\{\left(\mathbf{A}_{1}, \mathbf{B}_{1}, \mathbf{C}_{1}\right),\left(\mathbf{A}_{2}, \mathbf{B}_{2}, \mathbf{C}_{2}\right), \ldots,\left(\mathbf{A}_{q}, \mathbf{B}_{q}, \mathbf{C}_{q}\right)\right\} \text {. }
$$

The matrices $\Delta \mathbf{A}(i), i \in S$, and $\Delta \mathbf{B}(i), i \in S$, are uncertain system matrices and uncertain input matrices. They can be, respectively, denoted as

$$
\begin{array}{ll}
\Delta \mathbf{A}(i)=\mathbf{E}_{1} \boldsymbol{\Gamma}(i) \mathbf{F}_{1}, & i \in S, \\
\Delta \mathbf{B}(i)=\mathbf{E}_{2} \mathbf{T}(i) \mathbf{F}_{2}, & i \in S .
\end{array}
$$

Here, the matrices pairs $\left(\mathbf{E}_{1}, \mathbf{F}_{1}\right)$ and $\left(\mathbf{E}_{2}, \mathbf{F}_{2}\right)$ which characterize the structures of the system uncertainties and the input uncertainties are known real constant matrices with 
appropriate dimensions. The matrices $\Gamma(i)$ and $\mathbf{T}(i)$ are unknown but satisfy that $\boldsymbol{\Gamma}^{\mathrm{T}}(i) \boldsymbol{\Gamma}(i)-\mathbf{I}$ and $\mathbf{T}^{\mathrm{T}}(i) \mathbf{T}(i)-\mathbf{I}$ are negative definite, where $\mathbf{I}$ denotes an identity matrix with appropriate dimension. For description convenience, $\Delta \mathbf{A}(i)$ and $\Delta \mathbf{B}(i)$ are uniformly denoted as model uncertainties. The symbols $m_{k+1}(i) \in \mathbb{R}$ and $n_{k+1}(i+1) \in \mathbb{R}$ are load noise and measurement noise, respectively.

Given a desired trajectory $y_{d}(i+1), i \in S$, the objective of an ILC strategy for system (1) is to generate an input sequence $\left\{u_{k}(i), i \in S, k \in \mathbb{Z}_{+}\right\}$in a recursive mode by learning from the previous experience such that it can drive the output of system (1) to track the desired trajectory $y_{d}(i+1), i \in S$, as precisely as possible as iteration number tends to infinity. Mathematically, the inequality

$$
\lim _{k \rightarrow \infty} \sup \left|e_{k+1}(i+1)\right| \leq \varepsilon, \quad \forall i \in S
$$

is true for a sufficiently small positive number $\varepsilon$, where $e_{k}(i+$ $1)=y_{d}(i+1)-y_{k}(i+1)$ is termed as the tracking error and "lim sup" stands for the supremum limit of a sequence.

In this paper, a PD-type ILC scheme is considered as

$$
u_{k+1}(i)=u_{k}(i)+\gamma_{\tau(i)} e_{k}(i+1)+\kappa_{\tau(i)} e_{k}(i), \quad i \in S,
$$

where $\gamma_{\tau(i)}$ and $\kappa_{\tau(i)}$ denote the differential gains and the proportional gains, respectively.

The subsequent analysis of this paper is based on the following assumptions.

Assumption 1. Every operation starts at the same initial state; that is, $\mathbf{x}_{k+1}(0)=\mathbf{x}_{0}, \forall k \in \mathbb{N}_{+}$. Without loss of generality, it is assumed that $\mathbf{x}_{0}=\mathbf{0}$.

Assumption 2. The desired trajectory $y_{d}(i+1), i \in S$, is iteration-invariant.

Assumption 3. For an arbitrary trajectory $y_{d}(i+1), i \in S$, there exist a desired control input signal $u_{d}(i), i \in S$, and a desired state vector $\mathbf{x}_{d}(i), i \in S$, such that

$$
\begin{aligned}
& \mathbf{x}_{d}(i+1)=\mathbf{A}_{\tau(i)} \mathbf{x}_{d}(i)+\mathbf{B}_{\tau(i)} u_{d}(i), \\
& y_{d}(i+1)=\mathbf{C}_{\tau(i)} \mathbf{x}_{d}(i+1),
\end{aligned}
$$

$i \in S$.

Note that Assumption 3 is basic for the subsequent theoretical analysis. First of all, it is a solvable condition of the switched system (1). If this assumption is not satisfied the following discussions will be worthless. In addition, it is concluded that, from Assumption 3, there uniquely exists a desired control input to drive the output of system (1) to asymptotically follow a given desired trajectory, theoretically.

Assumption 4. The switching rule remains iteration-invariant once it is arbitrarily specified at the first trial.

The learning performance of the ILC law (5) will be analyzed by employing the super-vector method. It is necessary to rewrite the original systems (1) as follows.
Given $N-1 \in S$, the outputs of system (1) from the time instant 1 to $N$ at the $(k+1)$ th trial can be, respectively, derived as

$$
\begin{aligned}
& y_{k+1}(1)=\mathbf{C}_{\tau(0)} \mathbf{x}_{k+1}(1)=\mathbf{C}_{\tau(0)} \widehat{\mathbf{A}}_{\tau(0)} \mathbf{x}_{k+1}(0) \\
& +\mathbf{C}_{\tau(0)} \widehat{\mathbf{B}}_{\tau(0)} u_{k+1}(0)+\mathbf{C}_{\tau(0)} \widehat{\mathbf{B}}_{\tau(0)} m_{k+1}(0)+n_{k+1}(1), \\
& y_{k+1}(2)=\mathbf{C}_{\tau(1)} \mathbf{x}_{k+1}(2)=\mathbf{C}_{\tau(1)} \widehat{\mathbf{A}}_{\tau(1)} \widehat{\mathbf{A}}_{\tau(0)} \mathbf{x}_{k+1}(0) \\
& +\mathbf{C}_{\tau(1)} \widehat{\mathbf{A}}_{\tau(1)} \widehat{\mathbf{B}}_{\tau(0)} u_{k+1}(0)+\mathbf{C}_{\tau(1)} \widehat{\mathbf{B}}_{\tau(1)} u_{k+1}(1) \\
& +\mathbf{C}_{\tau(1)} \widehat{\mathbf{A}}_{\tau(1)} \widehat{\mathbf{B}}_{\tau(0)} m_{k+1}(0)+\mathbf{C}_{\tau(1)} \widehat{\mathbf{B}}_{\tau(1)} m_{k+1}(1) \\
& +n_{k+1}(2) \text {, } \\
& y_{k+1}(3)=\mathbf{C}_{\tau(2)} \mathbf{x}_{k+1}(3)=\mathbf{C}_{\tau(2)} \widehat{\mathbf{A}}_{\tau(2)} \widehat{\mathbf{A}}_{\tau(1)} \widehat{\mathbf{A}}_{\tau(0)} \mathbf{x}_{k+1}(0) \\
& +\mathbf{C}_{\tau(2)} \widehat{\mathbf{A}}_{\tau(2)} \widehat{\mathbf{A}}_{\tau(1)} \widehat{\mathbf{B}}_{\tau(0)} u_{k+1}(0) \\
& +\mathbf{C}_{\tau(2)} \widehat{\mathbf{A}}_{\tau(2)} \widehat{\mathbf{B}}_{\tau(1)} u_{k+1}(1)+\mathbf{C}_{\tau(2)} \widehat{\mathbf{B}}_{\tau(2)} u_{k+1}(2) \\
& +\mathbf{C}_{\tau(2)} \widehat{\mathbf{A}}_{\tau(2)} \widehat{\mathbf{A}}_{\tau(1)} \widehat{\mathbf{B}}_{\tau(0)} m_{k+1}(0) \\
& +\mathbf{C}_{\tau(2)} \widehat{\mathbf{A}}_{\tau(2)} \widehat{\mathbf{B}}_{\tau(1)} m_{k+1}(1)+\mathbf{C}_{\tau(2)} \widehat{\mathbf{B}}_{\tau(2)} m_{k+1}(2) \\
& +n_{k+1}(2) \text {, } \\
& y_{k+1}(N)=\mathbf{C}_{\tau(N-1)} \mathbf{x}_{k+1}(N)=\mathbf{C}_{\tau(N-1)} \prod_{j=0}^{N-1} \widehat{\mathbf{A}}_{\tau(j)} \mathbf{x}_{k+1}(0) \\
& +\mathbf{C}_{\tau(i N-1)} \prod_{j=1}^{N-1} \widehat{\mathbf{A}}_{\tau(j)} \widehat{\mathbf{B}}_{\tau(0)} u_{k+1}(0) \\
& +\mathbf{C}_{\tau(N-1)} \prod_{j=2}^{N-1} \widehat{\mathbf{A}}_{\tau(j)} \widehat{\mathbf{B}}_{\tau(1)} u_{k+1}(1)+\cdots \\
& +\mathbf{C}_{\tau(N-1)} \widehat{\mathbf{A}}_{\tau(N-1)} \widehat{\mathbf{B}}_{\tau(N-2)} u_{k+1}(N-2) \\
& +\mathbf{C}_{\tau(i)} \widehat{\mathbf{B}}_{\tau(i)} u_{k+1}(N-1) \\
& +\mathbf{C}_{\tau(N-1)} \prod_{j=1}^{N-1} \widehat{\mathbf{A}}_{\tau(j)} \widehat{\mathbf{B}}_{\tau(0)} m_{k+1}(0) \\
& +\mathbf{C}_{\tau(N-1)} \prod_{j=2}^{N-1} \widehat{\mathbf{A}}_{\tau(j)} \widehat{\mathbf{B}}_{\tau(1)} m_{k+1}(1)+\cdots \\
& +\mathbf{C}_{\tau(N-1)} \widehat{\mathbf{A}}_{\tau(N-1)} \widehat{\mathbf{B}}_{\tau(N-2)} m_{k+1}(N-2) \\
& +\mathbf{C}_{\tau(N-1)} \widehat{\mathbf{B}}_{\tau(i)} m_{k+1}(N-1)+n_{k+1}(N),
\end{aligned}
$$

where $\widehat{\mathbf{A}}_{\tau(i)}=\mathbf{A}_{\tau(i)}+\Delta \mathbf{A}(i), i \in S$, and $\widehat{\mathbf{B}}_{\tau(i)}=\mathbf{B}_{\tau(i)}+\Delta \mathbf{B}(i)$, $i \in S$, for expression simplicity. By denoting the super vectors as

$$
\begin{aligned}
\mathbf{u}_{k+1} & =\left[u_{k+1}(0), u_{k+1}(1), \ldots, u_{k+1}(N-1)\right]^{\mathrm{T}}, \\
\mathbf{y}_{k+1} & =\left[y_{k+1}(1), y_{k+1}(2), \ldots, y_{k+1}(N)\right]^{\mathrm{T}},
\end{aligned}
$$




$$
\begin{aligned}
\mathbf{m}_{k+1} & =\left[m_{k+1}(0), m_{k+1}(1), \ldots, m_{k+1}(N-1)\right]^{\mathrm{T}}, \\
\mathbf{n}_{k+1} & =\left[n_{k+1}(1), n_{k+1}(2), \ldots, n_{k+1}(N)\right]^{\mathrm{T}}
\end{aligned}
$$

system (7) can be equivalently represented as the following input-output response linear system:

$$
\mathbf{y}_{k+1}=\mathbf{v}+(\mathbf{H}+\Delta \mathbf{H}) \mathbf{u}_{k+1}+(\mathbf{H}+\Delta \mathbf{H}) \mathbf{m}_{k+1}+\mathbf{n}_{k+1} .
$$

Here,

$$
\begin{aligned}
\mathbf{v}= & {\left[\mathbf{C}_{\tau(0)} \widehat{\mathbf{A}}_{\tau(0)} x_{k+1}(0), \mathbf{C}_{\tau(1)} \prod_{j=0}^{1} \widehat{\mathbf{A}}_{\tau(j)}\right.} \\
& \cdot x_{k+1}(0), \mathbf{C}_{\tau(2)} \prod_{j=0}^{2} \widehat{\mathbf{A}}_{\tau(j)} x_{k+1}(0), \ldots, \mathbf{C}_{\tau(N-1)} \prod_{j=0}^{N-1} \widehat{\mathbf{A}}_{\tau(j)} \\
& \left.\cdot x_{k+1}(0)\right]^{\mathrm{T}}
\end{aligned}
$$

is the initial output super vector and

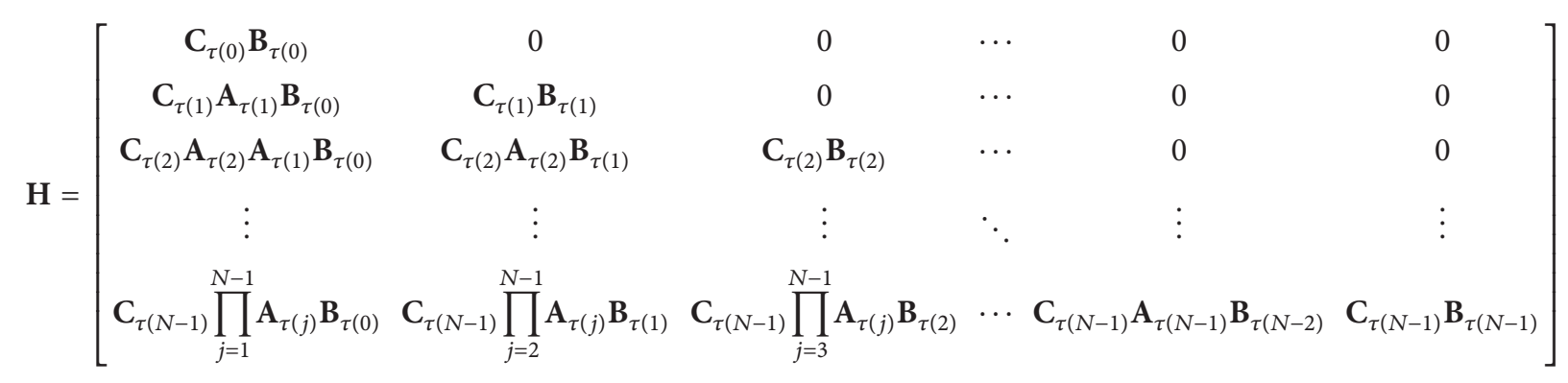

is a lifted matrix constructed by the nominal model of system (1) [21]. $\Delta \mathbf{H}$ is a lower triangle matrix which consists of the nominal model and the model uncertainties of systems (1). That is to say, $\Delta \mathbf{H}$ is also an uncertain matrix.

When taking Assumption 1 into account, the initial output super vector becomes $\mathbf{v}=\mathbf{0}$. Then system (9) becomes

$$
\mathbf{y}_{k+1}=(\mathbf{H}+\Delta \mathbf{H}) \mathbf{u}_{k+1}+(\mathbf{H}+\Delta \mathbf{H}) \mathbf{m}_{k+1}+\mathbf{n}_{k+1} .
$$

Thus, the control objective of ILC in the form of super vector can be equivalently described as searching such an input vector sequence $\left\{\mathbf{u}_{k}\right\}, k \in \mathbb{Z}_{+}$, that it can drive system (12) to track the desired trajectory $\mathbf{y}_{d}$ as precisely as possible as the iteration number tends to infinity. That is, the inequality

$$
\lim _{k \rightarrow \infty} \sup \left\|\mathbf{e}_{k+1}\right\|_{\infty} \leq \eta
$$

is satisfied for a small positive number $\eta$, where " $\|\cdot\|_{\infty}$ " denotes the infinite norm of a vector and $\mathbf{e}_{k+1}$ represents the tracking error super vector which is defined as

$$
\mathbf{e}_{k+1}=\mathbf{y}_{d}-\mathbf{y}_{k+1}=\left[e_{k+1}(1), e_{k+1}(2), \ldots, e_{k+1}(N)\right]^{\mathrm{T}},
$$

with a super-vector denotation of the desired trajectory $\mathbf{y}_{d}=$ $\left[y_{d}(1), y_{d}(2), \ldots, y_{d}(N)\right]^{\mathrm{T}}$.

In the framework of super vector, the updating law (5) is reformulated as

$$
\mathbf{u}_{k+1}=\mathbf{u}_{k}+\Phi \mathbf{e}_{k}
$$

where

$$
\boldsymbol{\Phi}=\left[\begin{array}{cccccc}
\gamma_{\tau(0)} & 0 & 0 & \cdots & 0 & 0 \\
\kappa_{\tau(1)} & \gamma_{\tau(1)} & 0 & \cdots & 0 & 0 \\
0 & \kappa_{\tau(2)} & \gamma_{\tau(2)} & \cdots & 0 & 0 \\
\vdots & \vdots & \vdots & \ddots & \vdots & \vdots \\
0 & 0 & 0 & \cdots & \kappa_{\tau(N-1)} & \gamma_{\tau(N-1)}
\end{array}\right]
$$

is the learning gain matrix which consists of the learning gains of the updating law (5). Here, $\kappa_{\tau(0)}$ is omitted, for $e_{k}(0)$ is not defined according to the specified operation time duration $S$. Now, the original two-dimensional ILC problem is converted into a one-dimensional linear inputoutput response problem [22].

\section{Main Results}

In order to access the learning performance of the PD-type ILC scheme (15) for the case when the controlled system (12) is disturbed by the model uncertainties and the external noises, the following sufficient condition of the monotone convergence of algorithm (15) is firstly deduced when both the model uncertainties and the external noises are null.

3.1. Convergence Analysis. If both the model uncertainties and the external noises are absent, the controlled system (12) becomes the nominal system denoted as

$$
\mathbf{y}_{k+1}=\mathbf{H} \mathbf{u}_{k+1} .
$$

When the updating law (15) is applied to system (17), Theorem 5 can be proven. 
Theorem 5. Suppose that ILC algorithm (15) is imposed on system (17) specified by a deterministic switching sequence $\{\tau(i)\}, i \in S$. The tracking error converges monotonically to zero in the sense of infinite norm, if there is a proper learning gain matrix $\Phi$ such that the inequality

$$
\|\mathbf{I}-\mathbf{H} \Phi\|_{\infty}<1
$$

holds, where " $\|\mathbf{M}\|_{\infty}$ " denotes the infinite norm of matrix $\mathbf{M}$.

Proof. By the definition of the tracking error super vector, it is easy to derive that

$$
\mathbf{e}_{k+1}=\mathbf{y}_{d}-\mathbf{y}_{k+1}=\mathbf{y}_{d}-\mathbf{H}\left(\mathbf{u}_{k}+\Phi \mathbf{e}_{k}\right)=(\mathbf{I}-\mathbf{H} \Phi) \mathbf{e}_{k} .
$$

Taking infinite norm of both sides of (19) and using norm inequality yield

$$
\left\|\mathbf{e}_{k+1}\right\|_{\infty} \leq\|\mathbf{I}-\mathbf{H} \Phi\|_{\infty}\left\|\mathbf{e}_{k}\right\|_{\infty} .
$$

By the assumption $\|\mathbf{I}-\mathbf{H} \Phi\|_{\infty}<1$, it is immediately deduced that $\left\|\mathbf{e}_{k+1}\right\|_{\infty}<\left\|\mathbf{e}_{k}\right\|_{\infty}$, which means that the sequence $\left\{\left\|\mathbf{e}_{k}\right\|_{\infty}\right\}$ monotonically decreases. Further, the inequality relation $\left\|\mathbf{e}_{k+1}\right\|_{\infty} \leq\|\mathbf{I}-\mathbf{H} \Phi\|_{\infty}^{k}\left\|\mathbf{e}_{1}\right\|_{\infty}$ can be easily derived by recursion, which means that $\lim _{k \rightarrow \infty}\left\|\mathbf{e}_{k+1}\right\|_{\infty}=0$.

This completes the proof.

For statement convenience, it is denoted that $\rho_{1}=\| \mathbf{I}-$ $\mathbf{H} \Phi \|_{\infty}$. Obviously, the magnitude of $\rho_{1}$ is a quantification to assess the convergence speed. Thus, it is regarded as socalled "learning factor" which is specified in [20]. From the perspective of numerical analysis, the convergence speed can be improved by choosing a proper learning gain matrix $\Phi$ to reduce the magnitude of the learning factor $\rho_{1}$.

3.2. Analysis of Robust Monotone Convergence. When the model uncertainties are present while the external noises are absent, system (12) becomes

$$
\mathbf{y}_{k+1}=(\mathbf{H}+\Delta \mathbf{H}) \mathbf{u}_{k+1} .
$$

Here, the robust monotone convergence is defined as follows.

Definition 6 (see [23]). An ILC algorithm is robustly monotonically convergent with respect to a vector norm $\|\cdot\|$ and a set of model uncertainties if, for every choice of the model uncertainty within this set and for every choice of initial control signal, the tracking error norm $\left\|\mathbf{e}_{k}\right\|$ converges strictly monotonically to zero.

Theorem 7. Assume that the updating law (15) is used on system (21) orchestrated by a deterministic switching sequence $\{\tau(i)\}, i \in S$, and there exists a proper learning gain matrix $\Phi$ such that condition (18) is guaranteed. Algorithm (15) is robustly monotonically convergent in the sense of infinite norm if the uncertain matrix $\Delta \mathbf{H}$ satisfies

$$
\|\Delta \mathbf{H}\|_{\infty}<\frac{1-\|\mathbf{I}-\mathbf{H} \Phi\|_{\infty}}{\|\Phi\|_{\infty}} .
$$

Proof. By the definition of the tracking error super vector, it is deduced that

$$
\mathbf{e}_{k+1}=[\mathbf{I}-(\mathbf{H}+\Delta \mathbf{H}) \Phi] \mathbf{e}_{k}=[(\mathbf{I}-\mathbf{H} \Phi)-\Delta \mathbf{H} \Phi] \mathbf{e}_{k} .
$$

Taking infinite norm of both sides of (23) and using norm inequality get

$$
\left\|\mathbf{e}_{k+1}\right\|_{\infty} \leq\left(\|\mathbf{I}-\mathbf{H} \boldsymbol{\Phi}\|_{\infty}+\|\Delta \mathbf{H}\|_{\infty}\|\boldsymbol{\Phi}\|_{\infty}\right)\left\|\mathbf{e}_{k}\right\|_{\infty} .
$$

Denoting $\rho_{2}=\|\mathbf{I}-\mathbf{H} \boldsymbol{\Phi}\|_{\infty}+\|\Delta \mathbf{H}\|_{\infty}\|\boldsymbol{\Phi}\|_{\infty}$ and taking assumption (22) into account yield

$$
\begin{aligned}
\rho_{2} & =\|\mathbf{I}-\mathbf{H} \boldsymbol{\Phi}\|_{\infty}+\|\Delta \mathbf{H}\|_{\infty}\|\boldsymbol{\Phi}\|_{\infty} \\
& <\|\mathbf{I}-\mathbf{H} \boldsymbol{\Phi}\|_{\infty}+\frac{1-\|\mathbf{I}-\mathbf{H} \Phi\|_{\infty}}{\|\boldsymbol{\Phi}\|_{\infty}}\|\boldsymbol{\Phi}\|_{\infty}=1 .
\end{aligned}
$$

Thus it is immediately derived that $\left\|\mathbf{e}_{k+1}\right\|_{\infty} \leq \rho_{2}\left\|\mathbf{e}_{k}\right\|_{\infty}<$ $\left\|\mathbf{e}_{k}\right\|_{\infty}$, which implies that sequence $\left\{\left\|\mathbf{e}_{k}\right\|_{\infty}\right\}$ monotonically decreases. By recursion, it is further deduced that inequality $\left\|\mathbf{e}_{k+1}\right\|_{\infty} \leq \rho_{2}^{k}\left\|\mathbf{e}_{1}\right\|_{\infty}$ is true. This means that $\lim _{k \rightarrow \infty}\left\|\mathbf{e}_{k+1}\right\|_{\infty}=0$.

This completes the proof.

3.3. Robustness Analysis. When the external noises are present while the model uncertainties are absent, system (12) can be reformulated as

$$
\mathbf{y}_{k+1}=\mathbf{H u}_{k+1}+\mathbf{H m}_{k+1}+\mathbf{n}_{k+1} .
$$

Therefore, the evolution of the tracking error vector at adjacent trials can be deduced as

$$
\mathbf{e}_{k+1}=(\mathbf{I}-\mathbf{H} \Phi) \mathbf{e}_{k}+\mathbf{H}\left(\mathbf{m}_{k}-\mathbf{m}_{k+1}\right)+\left(\mathbf{n}_{k}-\mathbf{n}_{k+1}\right) .
$$

Robustness referred to in this paper is as follows.

For a given desired trajectory $\mathbf{y}_{d}$ and an appropriated beginning input $\mathbf{u}_{1}$, the output $\mathbf{y}_{k+1}, k \in \mathbb{N}$, of system (26) driven by algorithm (15) asymptotically falls into a small neighborhood of the desired trajectory $\mathbf{y}_{d}$ as the iteration number goes to infinity if the external noises $\mathbf{m}_{k+1}$ and $\mathbf{n}_{k+1}$ are bounded within admissible ranges. Mathematically, inequality $\lim _{k \rightarrow \infty}$ sup $\left\|\mathbf{e}_{k+1}\right\|_{\infty} \leq \eta$ holds for a positive number $\eta$.

Theorem 8. Suppose that (27) is generated by applying the updating law (15) to the switched system (26) specified by a deterministic switching sequence $\{\tau(i)\}, i \in S$, and there exists a proper learning gain matrix $\Phi$ such that assumption (18) is true. Inequality

$$
\lim _{k \rightarrow \infty} \sup \left\|\mathbf{e}_{k+1}\right\|_{\infty}<\eta
$$

holds if the external noises satisfy $\left\|\mathbf{m}_{l}\right\|_{\infty}=\alpha<+\infty$ and $\left\|\mathbf{n}_{l}\right\|_{\infty}=\beta<+\infty$ for any $l \in \mathbb{Z}_{+}$, where $\eta$ is a positive number while $\alpha$ and $\beta$ are constants. 
Proof. Denoting $\delta \mathbf{m}_{k}=\mathbf{m}_{k}-\mathbf{m}_{k+1}, \delta \mathbf{n}_{k}=\mathbf{n}_{k}-\mathbf{n}_{k+1}$, taking infinite norm of both sides of (27), and using norm inequality yield

$$
\begin{aligned}
\left\|\mathbf{e}_{k+1}\right\|_{\infty} \leq & \left\|(\mathbf{I}-\mathbf{H} \Phi) \mathbf{e}_{k}\right\|_{\infty}+\left\|\mathbf{H} \delta \mathbf{m}_{k}\right\|_{\infty}+\left\|\delta \mathbf{n}_{k}\right\|_{\infty} \\
\leq & \|\mathbf{I}-\mathbf{H} \Phi\|_{\infty}\left\|\mathbf{e}_{k}\right\|+\|\mathbf{H}\|_{\infty}\left\|\delta \mathbf{m}_{k}\right\|_{\infty} \\
& +\left\|\delta \mathbf{n}_{k}\right\|_{\infty} .
\end{aligned}
$$

The assumptions $\left\|\mathbf{m}_{k}\right\|_{\infty}=\alpha<+\infty$ and $\left\|\mathbf{n}_{k}\right\|_{\infty}=\beta<$ $+\infty$ give that the relations $\left\|\delta \mathbf{m}_{k}\right\|_{\infty} \leq 2 \alpha,\left\|\delta \mathbf{n}_{k}\right\|_{\infty} \leq 2 \beta$ are satisfied. This means that the relations sup $\left\|\delta \mathbf{m}_{k}\right\|_{\infty} \leq 2 \alpha$ and $\sup \left\|\delta \mathbf{n}_{k}\right\|_{\infty} \leq 2 \beta$ hold.

Thus, it is further deduced that, by recursion inference,

$$
\begin{aligned}
& \left\|\mathbf{e}_{k+1}\right\|_{\infty} \leq\|\mathbf{I}-\mathbf{H} \Phi\|_{\infty}\left\|\mathbf{e}_{k}\right\|_{\infty}+2\left(\alpha\|\mathbf{H}\|_{\infty}+\beta\right) \\
& \leq\|\mathbf{I}-\mathbf{H} \Phi\|_{\infty}^{2}\left\|\mathbf{e}_{k-1}\right\|_{\infty} \\
& \quad+2\left(\alpha\|\mathbf{H}\|_{\infty}+\beta\right)\left(\|\mathbf{I}-\mathbf{H} \Phi\|_{\infty}+1\right) \\
& \leq\|\mathbf{I}-\mathbf{H} \Phi\|_{\infty}^{3}\left\|\mathbf{e}_{k-2}\right\|_{\infty} \\
& \quad+2\left(\alpha\|\mathbf{H}\|_{\infty}+\beta\right)\left(\|\mathbf{I}-\mathbf{H} \Phi\|_{\infty}^{2}+\|\mathbf{I}-\mathbf{H} \Phi\|_{\infty}+1\right) \\
& \quad \vdots \\
& \leq\|\mathbf{I}-\mathbf{H} \Phi\|_{\infty}^{k}\left\|\mathbf{e}_{1}\right\|_{\infty} \\
& \quad+2\left(\alpha\|\mathbf{H}\|_{\infty}+\beta\right) \sum_{l=0}^{k-1}\|\mathbf{I}-\mathbf{H} \Phi\|_{\infty}^{l} .
\end{aligned}
$$

When denoting $\rho_{1}=\|\mathbf{I}-\mathbf{H} \Phi\|_{\infty}$, inequality (30) is rewritten as

$$
\left\|\mathbf{e}_{k+1}\right\|_{\infty} \leq \rho_{1}^{k}\left\|\mathbf{e}_{1}\right\|_{\infty}+2\left(\alpha\|\mathbf{H}\|_{\infty}+\beta\right) \frac{1-\rho_{1}^{k}}{1-\rho_{1}} .
$$

Assumption $\rho_{1}<1$ leads to the fact that inequality

$$
\frac{1-\rho_{1}^{k}}{1-\rho_{1}}<\frac{1-\rho_{1}^{k+1}}{1-\rho_{1}}<\frac{1}{1-\rho_{1}}
$$

holds. That is to say, the sequence $\left\{\left(1-\rho_{1}^{k}\right) /\left(1-\rho_{1}\right)\right\}_{k=1}^{+\infty}$ monotonically increases and has upper bound. Thus the limit $\lim _{k \rightarrow+\infty}\left(1-\rho_{1}^{k}\right) /\left(1-\rho_{1}\right)$ exists. Taking supremum limit of both sides of (31) gets

$$
\begin{aligned}
\lim _{k \rightarrow \infty} \sup \left\|\mathbf{e}_{k+1}\right\|_{\infty} \leq & \lim _{k \rightarrow \infty} \rho_{1}^{k}\left\|\mathbf{e}_{1}\right\|_{\infty} \\
& +2\left(\alpha\|\mathbf{H}\|_{\infty}+\beta\right) \lim _{k \rightarrow \infty} \frac{1-\rho_{1}^{k}}{1-\rho_{1}} \\
& =\frac{2\left(\alpha\|\mathbf{H}\|_{\infty}+\beta\right)}{1-\rho_{1}} .
\end{aligned}
$$

By denoting $\eta=2\left(\alpha\|\mathbf{H}\|_{\infty}+\beta\right) /\left(1-\rho_{1}\right)$, inequality (28) is immediately deduced.

This completes the proof.
It is evident that, by inequality (33), the output of system (26) disturbed by the bounded external noises falls into a neighborhood of the desired trajectory $\mathbf{y}_{d}$ when PD-type ILC algorithm (15) is used. That is, algorithm (15) is robust against the bounded external noises. Also, it is seen that the robustness of algorithm (15) can be improved by choosing a proper learning gain matrix $\Phi$ to reduce the magnitude of $\rho_{1}$ if the boundaries of the external noises are constant.

Remark 9. The robust analysis in Theorem 8 is based on a situation that the system uncertainties are absent. Obviously, there exists another situation where the controlled system is perturbed by both the model uncertainties and the external noises. Actually, in this case, analogous results can be deduced when the model uncertainties are time-varying and satisfy assumption (22), as the value of $\rho_{1}$ in Theorem 8 can be replaced by that of $\rho_{2}$.

Remark 10. Compared with the existing ILC algorithms for the switched systems in $[3,13,18,19]$, this paper analyzes the robust monotone convergence when the model uncertainties are present and the robustness against the bounded external noises by employing super-vector method. The results are deduced under the condition that the system dynamics is periodically resettable and the switching rules are arbitrarily prespecified and iteration-invariant. Although the paper focuses on a class of time-invariant switched systems, the analysis implies that the proposed ILC algorithm may work well for linear time-varying switched systems specified by the deterministic time-dependent switching rules.

Remark 11. It is seen that, from the mechanism of ILC, the ILC strategy is an error-driven control strategy. The tracking error information of historical iterations is solely significant for updating the control input. This implies that the activated subsystems running over a certain time subinterval of different iterations are identical. Thus, the switching rules are required to be prespecified and iteration-invariant. Actually, the PD-type ILC algorithm in the paper is proposed on basis that the switching rules are arbitrarily prespecified and preknown. If the switching rules are nonpreset and unknown, it cannot be guaranteed that the subsystems running over the certain time subinterval of different trials are the same. Thus it is difficult to analyze the learning performance of the proposed ILC algorithm. However, if the switching rules are preset and unknown, the proposed PD-type ILC algorithm will work well. In this case, the learning performance of the algorithm can be analyzed from the perspective of stability. In the future work, this issue will be further addressed.

Remark 12. It is necessary to point out that the study on the ILC algorithms for the switched systems is more challenging than the study for the nonswitched systems. Firstly, as seen that a switched system consists of different subsystems specified by the switching rules, the learning process requires much more memory to store the learning gains, the potential dynamics of subsystems, and the switching rules. It would be a dimensional disaster if the system dimension is higher and the sampling number is much larger. However, the memory 


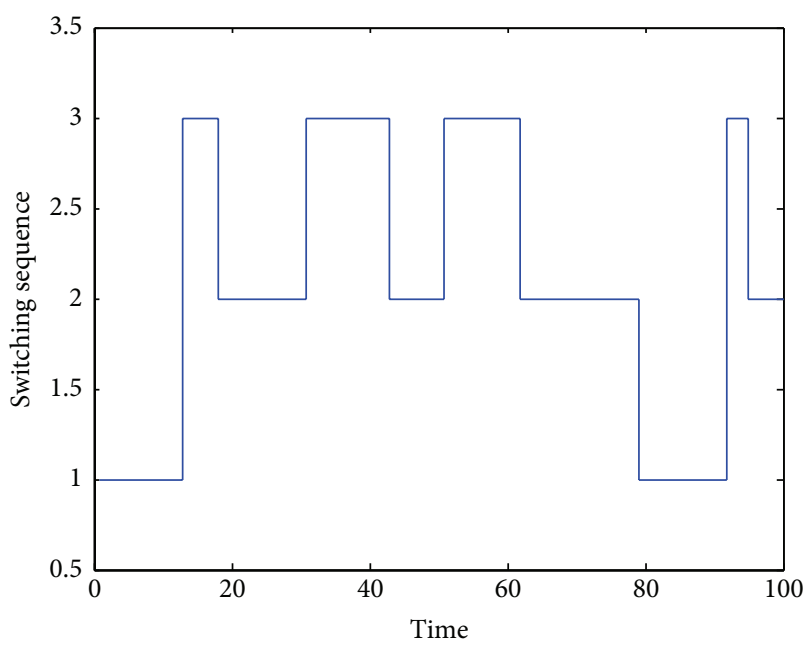

FIGURE 1: Switching rule $\tau(i)$.

requirement will be solved sooner or later with advancing of the information technology. Secondly, the switching rule involved in this paper is time-dependent and prespecified. However, there exist other types of switching rules in practice, such as state-dependent switching rules, event-dependent switching rules, and autonomous switching rules [2]. How to analyze the learning performance of the ILC algorithms for the switched systems specified by these switching rules is also difficult. Now it is still a pendent question but, simultaneously, is also an interesting consideration from an application point of view.

\section{Numerical Simulations}

To manifest the feasibility and the effectiveness of the proposed updating law (5) or (15), a simple example is considered. The controlled switched system is formulated as (1), where the operation discrete-time duration is set as $S=\{0,1,2, \ldots, 99\}$ (i.e., $N=100$ ). Assume that the switching sequence is specified as Figure 1 which shows that the controlled system consists of three subsystems.

Thus, system (1) with three subsystems is reformulated as

$$
\begin{aligned}
\mathbf{x}_{k+1}(i+1)= & \left(\mathbf{A}_{q}+\Delta \mathbf{A}(i)\right) \mathbf{x}_{k+1}(i) \\
& +\left(\mathbf{B}_{q}+\Delta \mathbf{B}(i)\right)\left(u_{k+1}(i)+m_{k+1}(i)\right), \\
y_{k+1}(i+1)= & \mathbf{C}_{q} \mathbf{x}_{k+1}(i+1)+n_{k+1}(i+1), \\
& \quad i \in S, q=1,2,3 .
\end{aligned}
$$

Here, the nominal dynamics is given as

$$
\begin{aligned}
& \mathbf{A}_{1}=\left[\begin{array}{cc}
0 & 1 \\
0.125 & -0.2
\end{array}\right], \\
& \mathbf{B}_{1}=\left[\begin{array}{l}
0 \\
1
\end{array}\right], \\
& \mathbf{C}_{1}=\left[\begin{array}{ll}
0.1 & 1
\end{array}\right],
\end{aligned}
$$

$$
\begin{aligned}
& \mathbf{A}_{2}=\left[\begin{array}{cc}
-0.25 & 1 \\
0 & -0.3
\end{array}\right], \\
& \mathbf{B}_{2}=\left[\begin{array}{l}
0 \\
1
\end{array}\right], \\
& \mathbf{C}_{2}=\left[\begin{array}{ll}
-0.2 & 1
\end{array}\right], \\
& \mathbf{A}_{3}=\left[\begin{array}{cc}
1 & 0 \\
0.2 & -0.1
\end{array}\right], \\
& \mathbf{B}_{3}=\left[\begin{array}{l}
0 \\
1
\end{array}\right], \\
& \mathbf{C}_{3}=\left[\begin{array}{ll}
0.25 & 1
\end{array}\right],
\end{aligned}
$$

which has served as an example in [3]. The model uncertainties $\Delta \mathbf{A}(i), \Delta \mathbf{B}(i)$ and the external noises $m_{k+1}(i), n_{k+1}(i)$, $i \in S$, are unknown but are bounded with admissible ranges. Denoted by the super-vector method, system (34) can be rewritten as

$$
\mathbf{y}_{k+1}=(\mathbf{H}+\Delta \mathbf{H}) \mathbf{u}_{k+1}+(\mathbf{H}+\Delta \mathbf{H}) \mathbf{m}_{k+1}+\mathbf{n}_{k+1} .
$$

Assume that the desired trajectory is selected as $y_{d}(i+$ $1)=\sin (2 i / 25) i \in S$. The initial state is set as $\mathbf{x}_{k+1}(0)=\mathbf{0}$ for all $k \in \mathbb{N}$ and the starting input vector is chosen as $\mathbf{u}_{1}(0)=$ 0. Throughout the implement, the differential gain $\gamma_{\tau(i)}$ and the proportional gain $\kappa_{\tau(i)}$ are assumed to be time-invariant and are set as $\gamma_{\tau(i)}=\gamma=0.85, \kappa_{\tau(i)}=\kappa=0.15$, respectively. In this case, the sufficient condition $\rho_{1}=\|\mathbf{I}-\mathbf{H} \Phi\|_{\infty}=0.7728<$ 1 is satisfied.

Note that, in Section 3, Theorems 5, 7, and 8 are established under the condition that the iteration number $k$ tends to infinity. However, it is hard to be implemented. In the simulations, the iteration number is set as $k=20$ to roughly verify the theoretical results derived in the above section.

4.1. Convergence. When the updating law (15) is applied to system (36) with neither the model uncertainties nor the external noises, the results of simulation are shown in Figures 2 and 3. Figure 2 exhibits the curve of $\left\|\mathbf{e}_{k}\right\|_{\infty}$ from the 1st trial to the 20th one. It is seen that $\left\|\mathbf{e}_{k}\right\|_{\infty}$ monotonically reduces to zero as iteration number enlarges. Figure 3 manifests the system outputs at the 3 rd trial and the 8 th trial, respectively. It is obvious that the output of the switched system (36) driven by the PD-type ILC algorithm (15) can track the desired trajectory, asymptotically. These illustrate that ILC algorithm (15) is valid if both the model uncertainties and the external noises are null.

4.2. Robust Monotone Convergence. For the case when the model uncertainties are present but the external noises are 


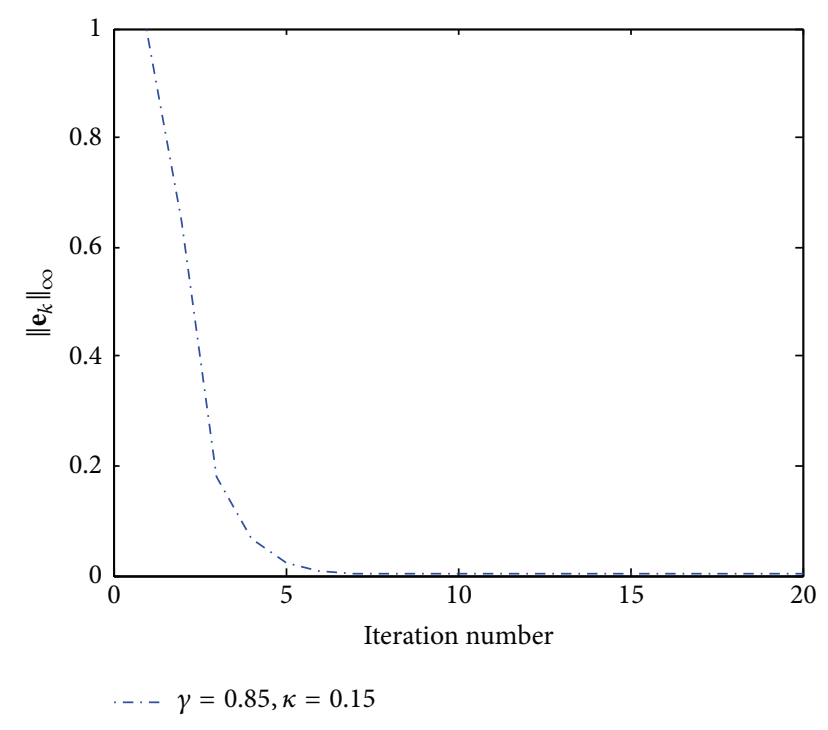

FIgURE 2: Tracking error when both model uncertainties and external noises are null.

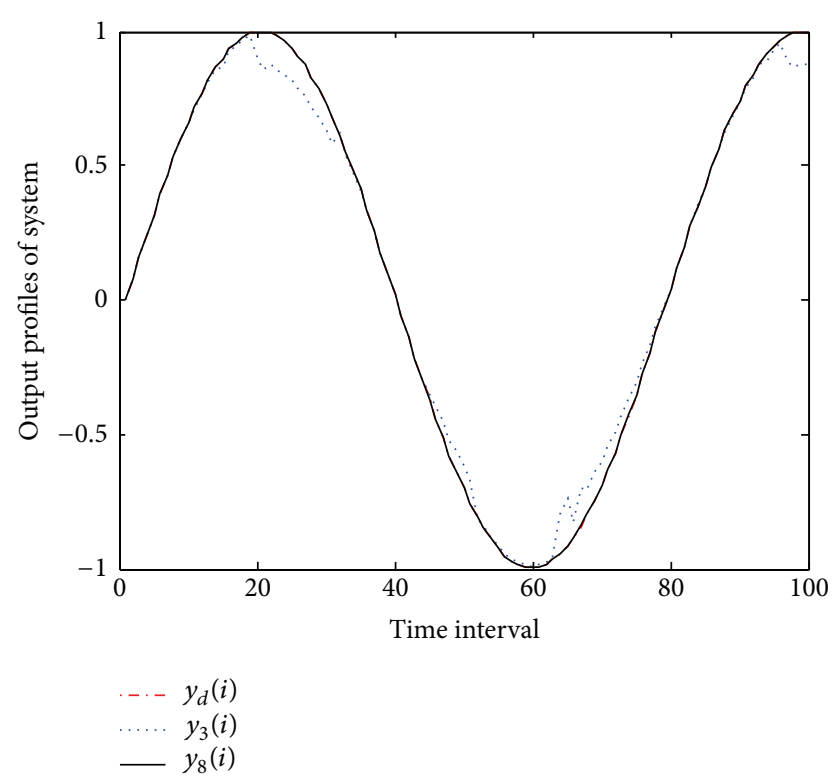

FIgURE 3: Outputs of the nominal system at the 3 rd trial and the 8th trial.

absent, the matrices pairs $\left(\mathbf{E}_{1}, \mathbf{F}_{1}\right)$ and $\left(\mathbf{E}_{2}, \mathbf{F}_{2}\right)$ are, respectively, set as

$$
\begin{aligned}
& \mathbf{E}_{1}=\left(\begin{array}{cc}
0.2 & 0 \\
0 & 0.2
\end{array}\right), \\
& \mathbf{F}_{1}=\left(\begin{array}{ll}
1 & 0 \\
0 & 1
\end{array}\right), \\
& \mathbf{E}_{2}=\left(\begin{array}{cc}
0.1 & 0 \\
0 & 0.1
\end{array}\right), \\
& \mathbf{F}_{2}=\left(\begin{array}{l}
0 \\
1
\end{array}\right) .
\end{aligned}
$$

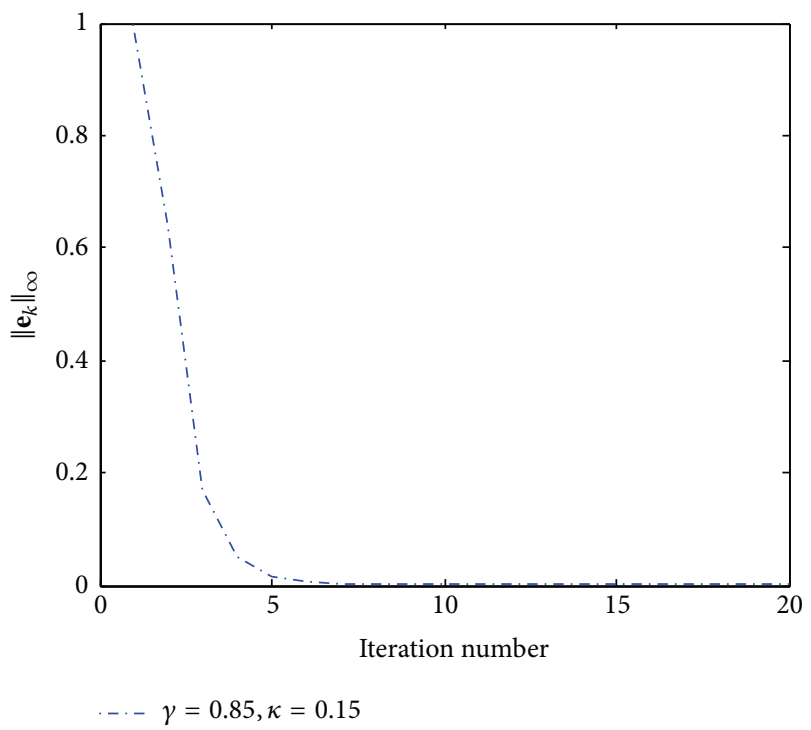

FIGURE 4: Tracking error when the controlled system is disturbed by model uncertainties.

Assume that the matrices $\boldsymbol{\Gamma}_{\tau(i)}$ and $\mathbf{T}_{\tau(i)}$ are considered as

$$
\Gamma_{\tau(i)}=\mathbf{T}_{\tau(i)}=\left(\begin{array}{cc}
\phi_{1}(i) & 0 \\
0 & \phi_{2}(i)
\end{array}\right) \text {, }
$$

where $\left|\phi_{j}(i)\right|<1, i \in S, j=1,2$. Based on (37) and (38), the uncertain matrix $\Delta \mathbf{H}$ is constructed. Randomly selecting two sequences $\left\{\phi_{1}(i)\right\}, i \in S$, and $\left\{\phi_{2}(i)\right\}, i \in S$, using the MATLAB function $\operatorname{rand}(\cdot)$, it is calculated that $\|\Delta \mathbf{H}\|_{\infty}=0.1429$. In addition, it is deduced that $\left(1-\|\mathbf{I}-\mathbf{H} \Phi\|_{\infty}\right) /\|\Phi\|_{\infty}=0.2272$ under the condition that the learning gains are chosen as $\gamma_{\tau(i)}=\gamma=0.85, \kappa_{\tau(i)}=\kappa=0.15$. Thus assumption (22) is satisfied.

The simulation results are shown in Figures 4 and 5 . Figure 4 manifests the convergence property of $\left\|\mathbf{e}_{k}\right\|_{\infty}$ generated by the PD-type ILC scheme (15). It is seen that $\left\|\mathbf{e}_{k}\right\|_{\infty}$ monotonically converges to zero when the controlled system is perturbed by the model uncertainties satisfying assumption (22). Figure 5 displays the outputs of system (36) at the 3 rd trial and the 8 th trial. It is evident that the output of the system stimulated by algorithm (15) asymptotically follows the desired trajectory when system (36) is only disturbed by the model uncertainties satisfying (22).

4.3. Robustness to External Noises. When the external bounded noises are present but the model uncertainties are absent, the controlled system (36) is denoted in the form of (26). Assume that the load noise vectors and the measurement noise vectors are generated by MATLAB functions $\mathbf{m}_{k+1}=0.04 \sin (\operatorname{randn}(N, 1))$ and $\mathbf{n}_{k+1}=0.04 \cos (\operatorname{randn}(N, 1))$. Since the effect of the load noises and the measurement noises is analogous, it is rational to merge two types of external noises as $\mathbf{w}_{k+1}=\mathbf{H} \xi_{k+1}+\varsigma_{k+1}$. The collective effect of the external noises at an arbitrary trial is illustrated in Figure 6.

The profile of $\left\|\mathbf{e}_{k}\right\|_{\infty}$ is shown in Figure 7 and the curve of $\max \left\{\left\|\mathbf{e}_{k}\right\|_{\infty}\right\}$ (instead of $\sup \left\{\left\|\mathbf{e}_{k}\right\|_{\infty}\right\}$ in Theorem 8) is 


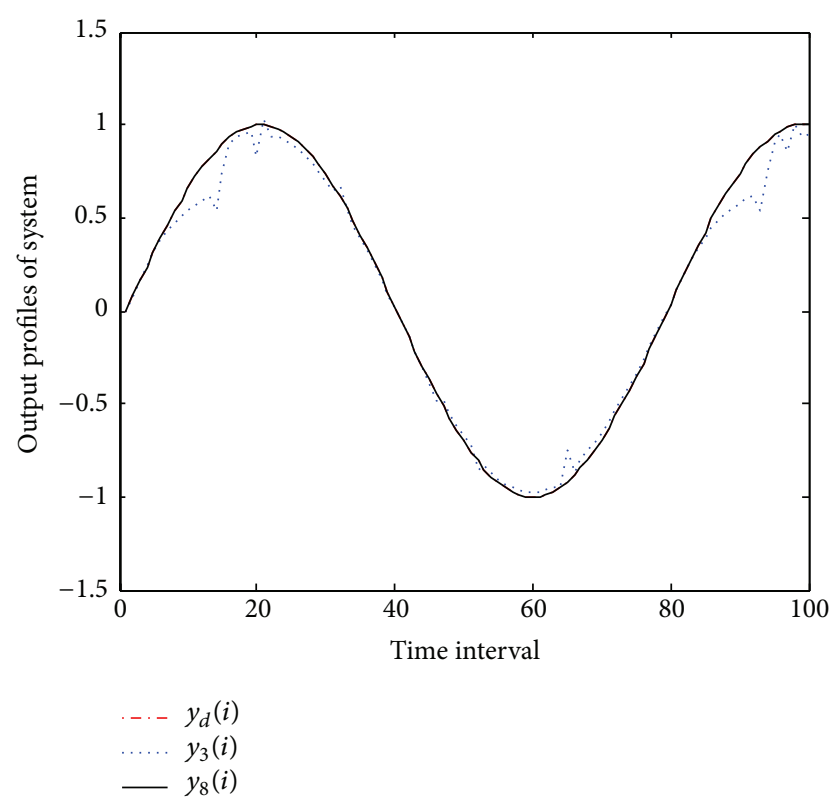

Figure 5: Outputs of system (36) at the 3rd trial and the 8th trial when the model uncertainties are present but the external noises are absent.

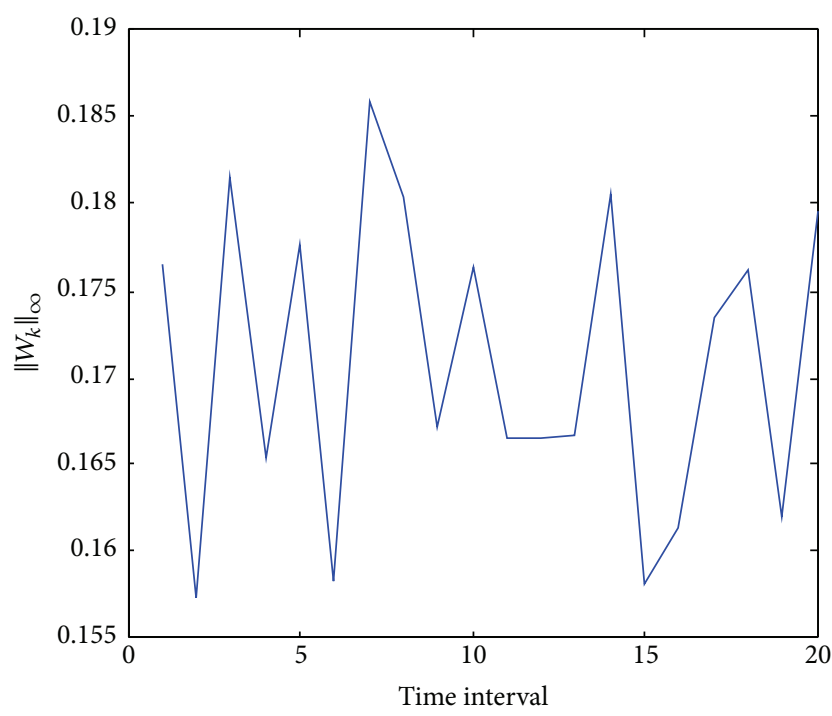

FIGURE 6: Collective effect of external noises at an arbitrary trial.

exhibited in Figure 8. It is obvious that the values of both $\left\|\mathbf{e}_{k}\right\|_{\infty}$ and $\max \left\{\left\|\mathbf{e}_{k}\right\|_{\infty}\right\}$ are bounded within an admissible range as the iteration number increases.

The outputs at the $3 \mathrm{rd}$ trial and the 8 th trial are illustrated in Figure 9. It is observed that the tracking performance of system (36) driven by the PD-type ILC scheme (15) is satisfactory when system (36) is only disturbed by external noises bounded within admissible ranges.

\section{Conclusion}

Assume that the switched systems repetitively operate over a finite time interval and the switching rules are arbitrarily

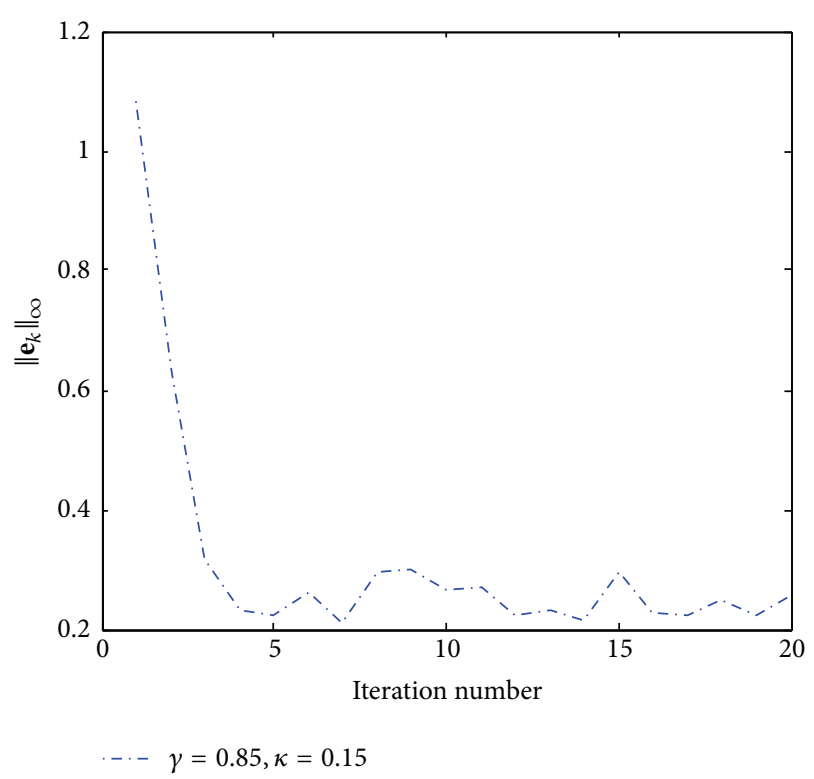

FIGURE 7: Tracking error when the controlled system is disturbed by the external noises.

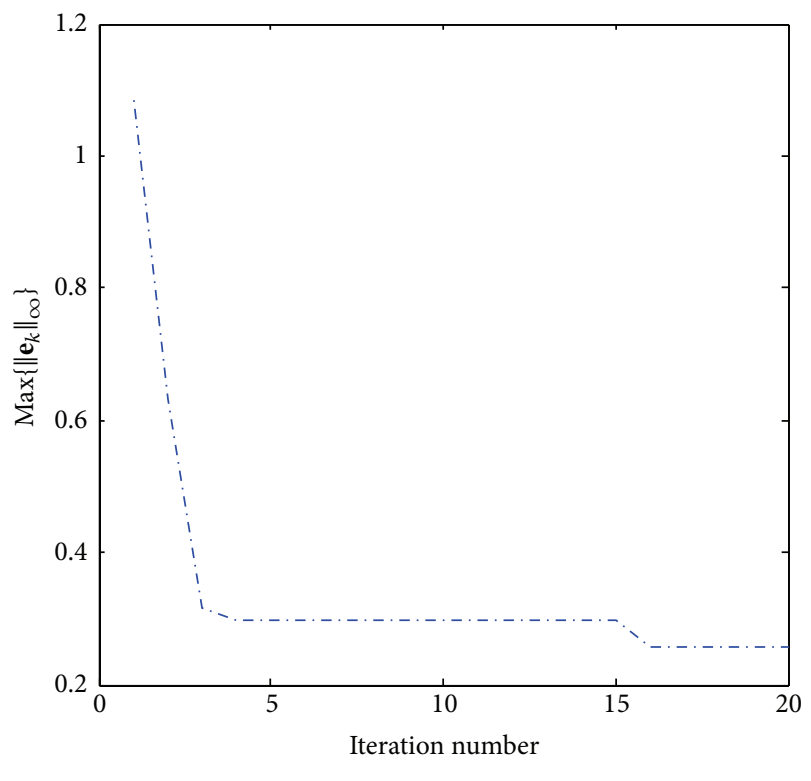

$$
\text { - - } \gamma=0.85, \kappa=0.15
$$

Figure 8: Curve of $\max \left\{\left\|\mathbf{e}_{k}\right\|_{\infty}\right\}$ which is the maximum of $\left\|\mathbf{e}_{k}\right\|_{\infty}$ $(k=1,2, \ldots, 20)$ in the last $(20-k+1)$ iterations.

prespecified. A PD-type ILC algorithm is imposed on a kind of discrete switched systems for tracking given trajectories. By employing the super-vector approach, a sufficient condition of monotone convergence is firstly deduced when the controlled switched system is nominal. Then the robust monotone convergence is analyzed for the case when the model uncertainties are present while the external noises are absent. Last, the robustness against the bounded external noises is discussed. Results manifest that the learning performance such as the convergence, the robust monotone convergence, 


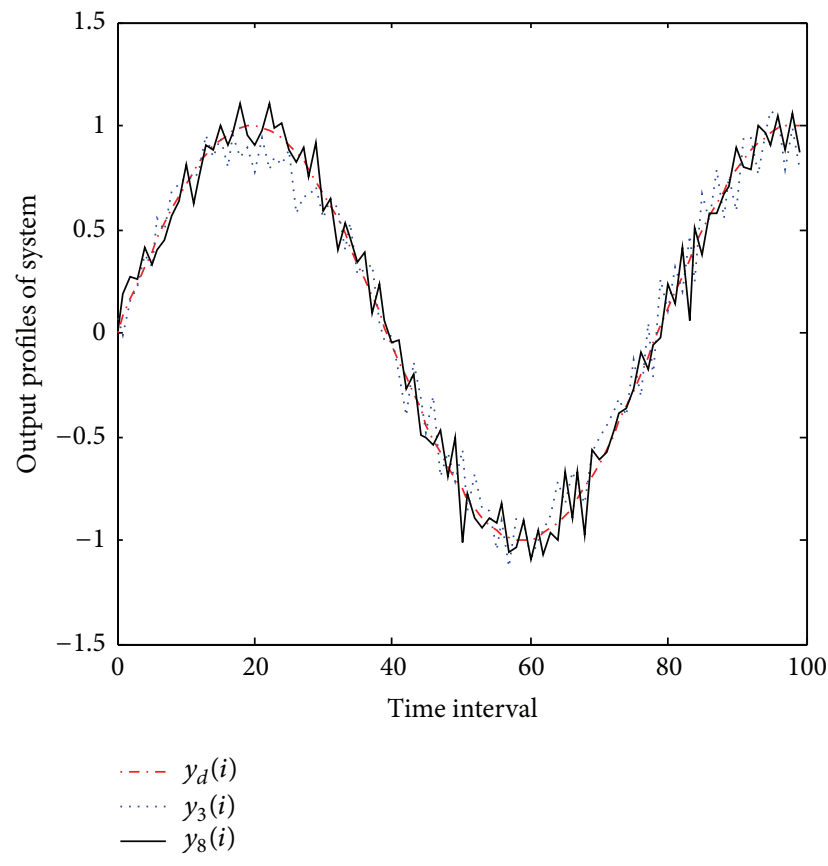

FIGURE 9: Outputs of system (36) at the 3rd trial and the 8th trial when the external noises are present while the model uncertainties are absent.

and the robustness can be guaranteed if the switching rules are time-variant and the related conditions are satisfied. The attached simulations support the fact that the proposed PDtype ILC algorithm is feasible and effective for the linear switched systems specified by the preset switching rules. However, when the switching rules are varying in the iterative domain, the learning performance analysis of the ILC scheme for the switched systems is a challenging topic.

\section{Conflict of Interests}

The author declares that there is no conflict of interests regarding the publication of this paper.

\section{References}

[1] M. de la Sen and A. Ibeas, "On the global asymptotic stability of switched linear time-varying systems with constant point delays," Discrete Dynamics in Nature and Society, vol. 2008, Article ID 231710, 31 pages, 2008.

[2] Z. Sun, Switched Linear Systems: Control and Design, Springer, 2006.

[3] X.-H. Bu, F.-S. Yu, Z.-S. Hou, and F.-Z. Wang, "Iterative learning control for a class of linear discrete-time switched systems," Acta Automatica Sinica, vol. 39, no. 9, pp. 1564-1569, 2013.

[4] L. Zhang, P. Shi, and M. Basin, "Robust stability and stabilization of uncertain switched linear discrete time-delay systems," IET Control Theory \& Applications, vol. 2, no. 7, pp. 606-614, 2008.
[5] J.-X. Lin and S.-M. Fei, "Robust exponential admissibility of uncertain switched singular time-delay systems," Acta Automatica Sinica, vol. 36, no. 12, pp. 1773-1779, 2010.

[6] J. Lian and J. Zhao, "Output feedback variable structure control for a class of uncertain switched systems," Asian Journal of Control, vol. 11, no. 1, pp. 31-39, 2009.

[7] H. Lin and P. J. Antsaklis, "Asymptotic disturbance attenuation properties for uncertain switched linear systems," Nonlinear Analysis: Hybrid Systems, vol. 4, no. 2, pp. 279-290, 2010.

[8] J. Li, W. Wu, J. Yuan, Q. Tan, and X. Yin, "Delay-dependent stability criterion of arbitrary switched linear systems with time-varying delay," Discrete Dynamics in Nature and Society, vol. 2010, Article ID 347129, 16 pages, 2010.

[9] B. Du and X. Zhang, "Delay-dependent stability analysis and synthesis for uncertain impulsive switched system with mixed delays," Discrete Dynamics in Nature and Society, vol. 2011, Article ID 381571, 9 pages, 2011.

[10] Z. Ji, L. Wang, and X. Guo, "Design of switching sequences for controllability realization of switched linear systems," Automatica, vol. 43, no. 4, pp. 662-668, 2007.

[11] D. Cheng, "Controllability of switched bilinear systems," IEEE Transactions on Automatic Control, vol. 50, no. 4, pp. 511-515, 2005.

[12] J. P. Hespanha, D. Liberzon, D. Angeli, and E. D. Sontag, "Nonlinear norm-observability notions and stability of switched systems," IEEE Transactions on Automatic Control, vol. 50, no. 2, pp. 154-168, 2005.

[13] X. Bu, Z. Hou, F. Yu, and Z. Fu, "Iterative learning control for a class of non-linear switched systems," IET Control Theory and Applications, vol. 7, no. 3, pp. 470-481, 2013.

[14] X. Xu and P. J. Antsaklis, "Optimal control of switched systems based on parameterization of the switching instants," IEEE Transactions on Automatic Control, vol. 49, no. 1, pp. 2-16, 2004.

[15] L. Wu, W. X. Zheng, and H. Gao, "Dissipativity-based sliding mode control of switched stochastic systems," IEEE Transactions on Automatic Control, vol. 58, no. 3, pp. 785-791, 2013.

[16] H. Song, L. Yu, D. Zhang, and W.-A. Zhang, "Finite-time $H_{\infty}$ control for a class of discrete-time switched time-delay systems with quantized feedback," Communications in Nonlinear Science and Numerical Simulation, vol. 17, no. 12, pp. 4802-4814, 2012.

[17] S. Arimoto, S. Kawamura, and F. Miyazaki, "Bettering operation of robots by learning," Journal of Robotic Systems, vol. 1, no. 2, pp. 123-140, 1984.

[18] X. Bu, F. Yu, Z. Fu, and F. Wang, "Stability analysis of highorder iterative learning control for a class of nonlinear switched systems," Abstract and Applied Analysis, vol. 2013, Article ID 684642, 13 pages, 2013.

[19] X. Bu, F. Yu, and Z. Hou, "Iterative learning control for linear switched systems with arbitrary switched rules," in Proceedings of the 10th World Congress on Intelligent Control and Automation (WCICA '12), pp. 1182-1187, Beijing, China, July 2012.

[20] X. Ruan, Z. Z. Bien, and Q. Wang, "Convergence properties of iterative learning control processes in the sense of the Lebesguep norm," Asian Journal of Control, vol. 14, no. 4, pp. 1095-1107, 2012.

[21] H.-S. Ahn, Y. Q. Chen, and K. L. Moore, "Iterative learning control: brief survey and categorization," IEEE Transactions on Systems, Man and Cybernetics Part C: Applications and Reviews, vol. 37, no. 6, pp. 1099-1121, 2007. 
[22] H. S. Ahn, K. L. Moore, and Y. Q. Chen, Iterative Learning Control: Robustness and Monotonic Convergence for Interval Systems, Springer, New York, NY, USA, 2007.

[23] J. Nygren, K. Pelckmans, and B. Carlsson, "Approximate adjoint-based iterative learning control," International Journal of Control, vol. 87, no. 5, pp. 1028-1046, 2014. 


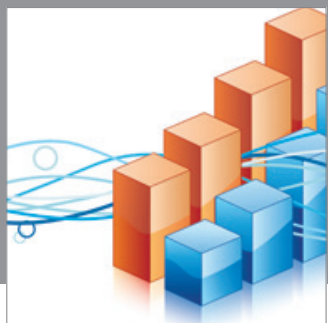

Advances in

Operations Research

mansans

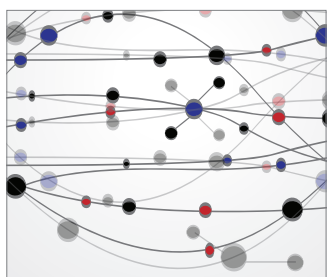

The Scientific World Journal
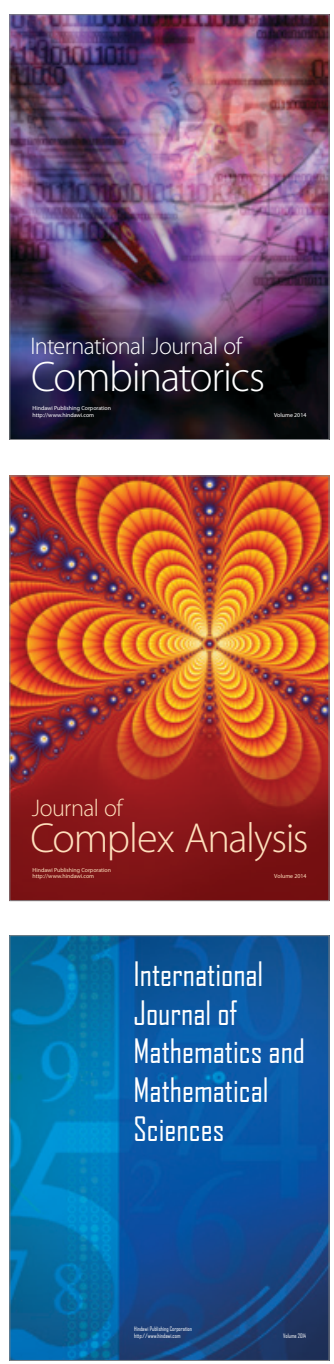
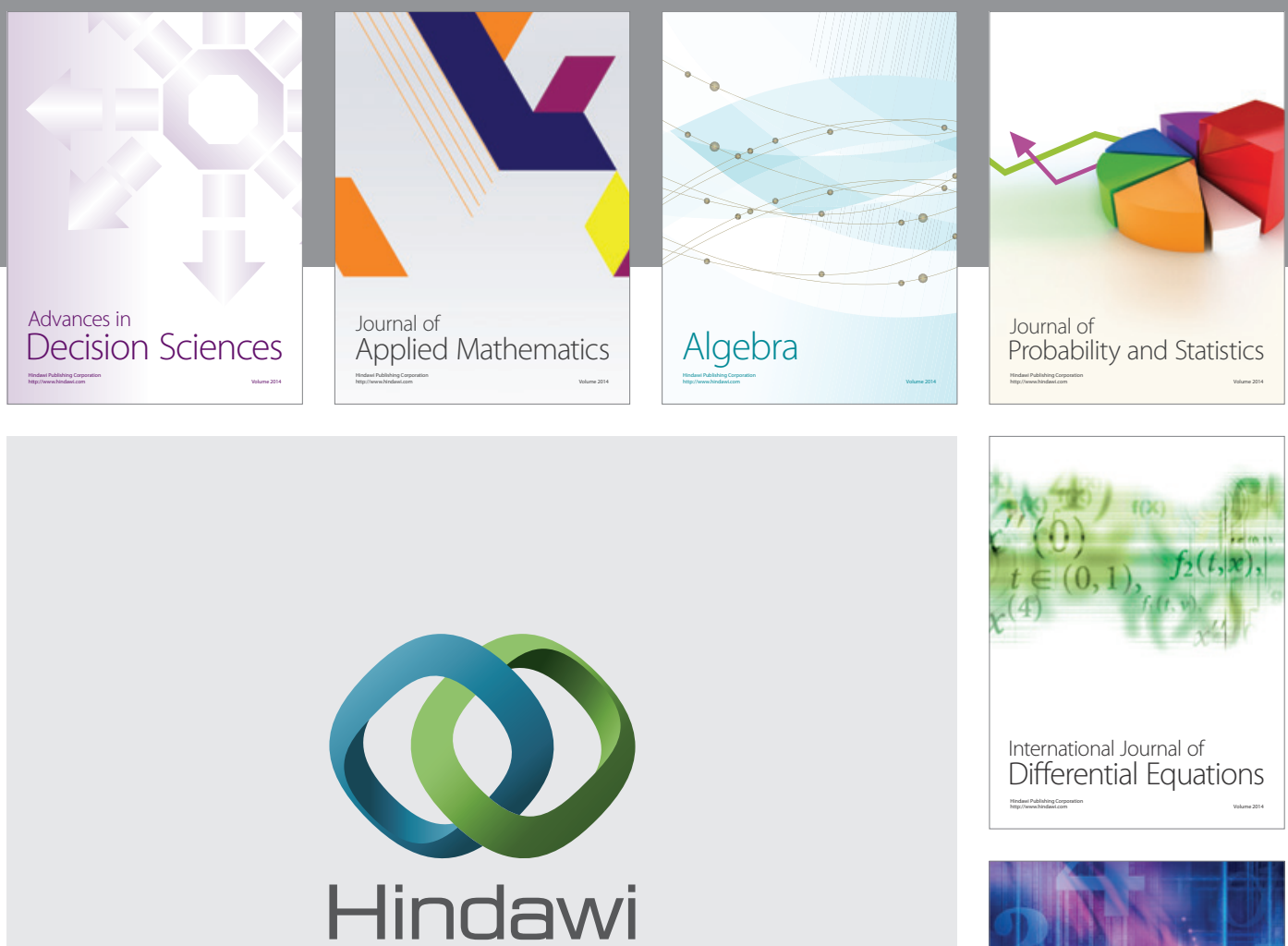

Submit your manuscripts at http://www.hindawi.com
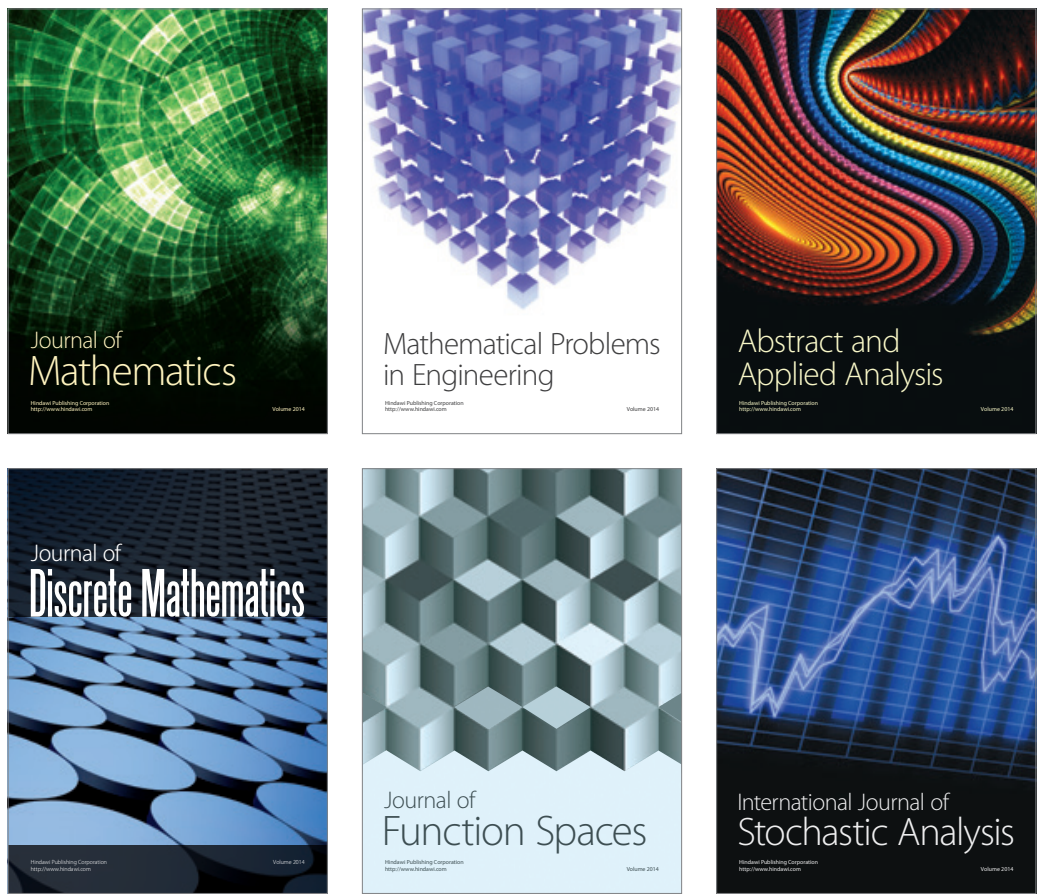

Journal of

Function Spaces

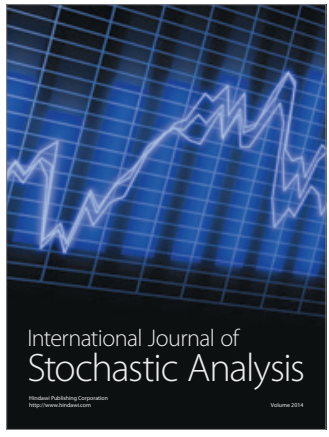

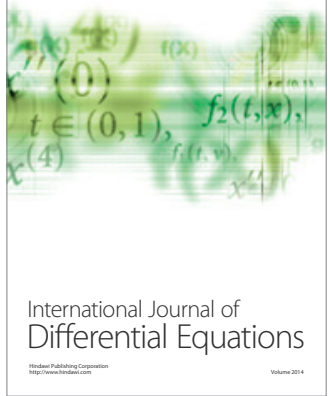
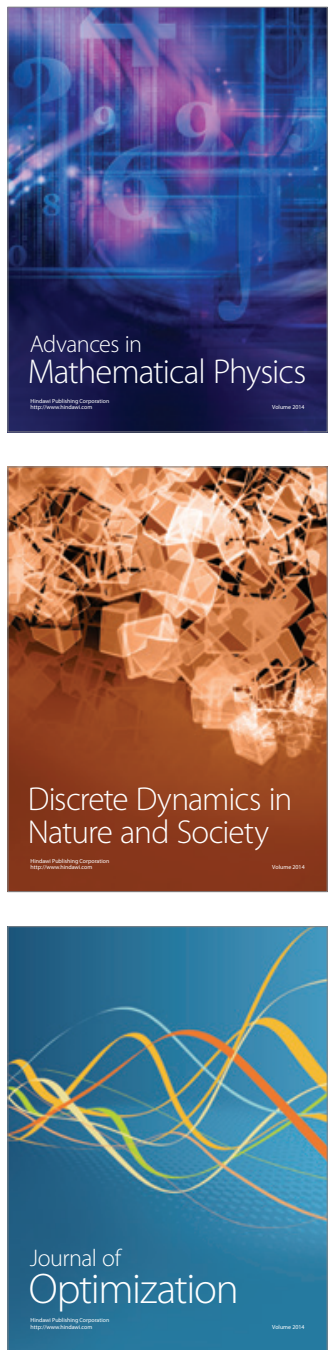